\title{
Oral anticoagulation and risk of death: a medical record linkage study
}

\author{
Anders Odén, Martin Fahlén
}

\begin{abstract}
Objective To study how mortality varies with different degrees of anticoagulation reflected by the international normalised ratio (INR).

Design Record linkage analysis with death hazard estimated as a continuous function of INR.

Data sources 46 anticoagulation clinics in Sweden with computerised medical records.

Subjects Records for 42451 patients, 3533 deaths, and 1.25 million INR measurements.

Main outcome measures Mortality from all causes and from intracranial haemorrhage.

Results Mortality from all causes of death was strongly related to level of INR. Minimum risk of death was attained at 2.2 INR for all patients and 2.3 INR for patients with mechanical heart valve prostheses. A high INR was associated with an excess mortality: with an increase of 1 unit of INR above 2.5, the risks of death from cerebral bleeding (149 deaths) and from any cause were about doubled. Among patients with an INR of $\geqslant 3.0,1069$ deaths occurred within 7 weeks; if the risk coincided with that with an INR of 2.9, the expected number of deaths would have been 569 . Thus at least 500 deaths were associated with a high INR value, but not necessarily caused by the treatment.

Conclusions The excess mortality associated with high INR values supports the use of less intensive treatment and a small therapeutic window, with INR close to 2.2-2.3 irrespective of the indication for anticoagulant treatment. More preventive actions should be taken to avoid episodes of high INR.
\end{abstract}

\section{Introduction}

Treatment with oral anticoagulants requires a constant balancing between undertreatment and overtreatment. The intensity of coagulation is monitored within narrow therapeutic margins. The effect of the daily dose has to be checked regularly because of the influence of disease, food, and other drugs on coagulation. It has been difficult to estimate the optimal degree of anticoagulation and how much it depends on the indication for treatment. Routines vary, but in recent years there has been a shift towards lower therapeutic ranges. ${ }^{1}$

The degree of anticoagulation is calculated from the prothrombin time and expressed as the international normalised ratio (INR). Different targets of INR for different conditions have been proposed, ${ }^{2}$ with more intense treatment at an INR of 2.5-3.5 (reference value for untreated patient is 1.0 ) recommended for patients with a mechanical heart valve prosthesis in the mitral position. ${ }^{3}$ Others find no reason to differentiate and suggest a target INR of 2.0-3.0 for all conditions. In order to minimise complications, a combination treatment of dipyridamole, aspirin, and a fixed low daily dose of warfarin has been suggested for patients with a heart valve prosthesis. ${ }^{4}$ Furthermore, the beneficial effect of anticoagulation after fitting a biological replacement for an aortic valve has been questioned.

With the introduction of computerised records at anticoagulation clinics it is possible to obtain data from much larger populations than have been used in clinical trials to improve our understanding of how to optimise treatment. We therefore analysed the medical records from about half of all anticoagulation clinics in Sweden.

\section{Methods}

Since 1985 the hospital based and centralised anticoagulation clinics in Sweden, where qualified nurses monitor anticoagulant treatment for outpatients with all types of conditions, have gradually become computerised. Using software made by Journalia, we analysed the records of 42451 patients ( $42 \%$ women) who attended 46 clinics during 1990-7, most of them towards the end of the period. Their mean age at the start of anticoagulation treatment was 70.5 years (SD 9.1 years). The main indications for anticoagulation were atrial fibrillation (58\%), venous thrombosis and pulmonary embolism (25\%), stroke and transient ischaemic attacks $(22 \%)$, valve prosthesis (18\%), and myocardial infarction $(3 \%)$. We identified patients' deaths from the registry of causes of death maintained by the Swedish National Board of Health and Welfare. This register includes more than $99 \%$ of all deaths in Sweden, and 3553 deaths contributed to our base analysis. The number of patient years was 60668 , and the number of INR measurements and anticoagulant doses used was 1.25 million. After one year of treatment, the mean interval between measurements was 18 days.

Our study was approved by the Ethics Committee for Medical Research at the University of Göteborg.

\section{Data analysis}

In our base analysis each patient was followed from one INR measurement to the next one or to death if either occurred within seven weeks. After this time, which is a common maximum interval between anticoagulant doses, we censored the patient data until a new INR measurement was performed, which was then used as a starting value for another period of surveillance. Thus, a large number of INR measurements were used as current INR values for estimating the death hazard function depending on age, sex, and INR. The hazard function was estimated by a Poisson model $^{7}$ and was continuous as a function of age and INR. The death hazard was $\exp \left(\beta_{0}+\beta_{1} \times x_{1}+1 / 4\right)$, where $\beta_{0}$, $\beta_{1}$, etc, were constants and $x_{1}, x_{2}$, etc, were the values of the variables. The logarithm of the hazard function was linear for INR values $<1.8$, quadratic between 1.8 and 2.5 , and linear for higher values. The corresponding $\beta$ values and their standard errors could be used to investigate the shape of the curve and to assess changes with INR. We performed separate analyses for the major indications for anticoagulation and analysed cerebral bleeding separately as a cause of death.
Valler 190, S-442 92
Romelanda,
Sweden
Anders Odén
consulting statistician
Department of
Medicine, Hospital
of Kungälv, Sweden
Martin Fahlén
associate professor of
medicine
Correspondence to:
A Odén
anders.oden@
mbox301.swipnet.se
BMJ 2002;325:1073-5 


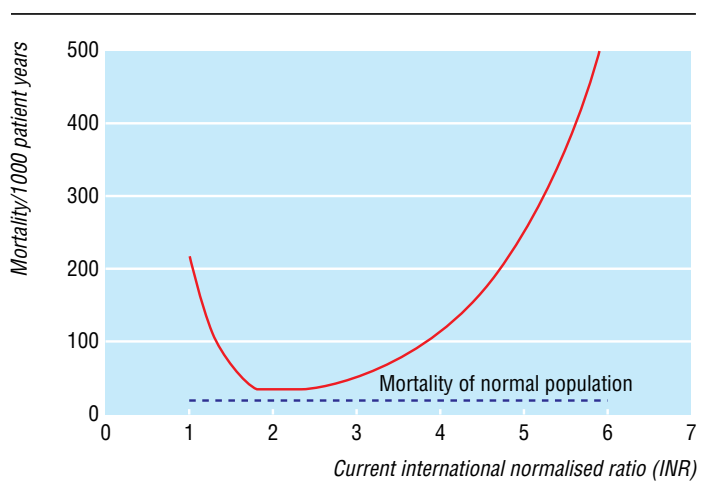

Risk of death associated with different levels of anticoagulation. The curve was calculated from the results of the Poisson model and shows, as an example, the risk for women aged 72: mean risk was 47.6 per 1000 patient years, attained roughly at INR values of 1.6 or 2.9

\section{Results}

The table shows the mortality associated with different INR values for all causes of death and for deaths where bleeding was a main or contributing cause. Poisson regression analysis allowed us to include age and sex as well as INR. There was a marked decrease in mortality from all causes with increasing INR in the interval 1-1.8 but also a substantial increase in mortality for INR values $>2.5$ (see figure). The death hazard function was lowest at an INR of 2.15. Eighteen per cent of INR values were $\geqslant 3.0$. For patients with mechanical heart valve prostheses (401 deaths), risk of death was lowest at an INR of 2.33. For other major indications for anticoagulation, the risk was lowest at INR 2.2. The risk increase per unit increase in INR above 2.5 was 2.2 (95\% confidence interval 2.1 to 2.2) for all indications, 2.1 for atrial fibrillation, 2.3 for venous thrombosis and pulmonary embolism, 2.3 for stroke and transient ischaemic attacks, and 2.1 for valve prosthesis.

We also calculated the risk of death from cerebral bleeding (ICD-9 codes 430, 431, and 432 and ICD-10 codes I60, I61, and I62). The risk significantly decreased with increasing INR in the INR interval 1-1.5 $(\mathrm{P}=0.002)$ and significantly increased at INR values of $\geqslant 1.5(\mathrm{P}<0.001)$. However, at values of $\geqslant 3.0$ the increased risk of dying from cerebral bleeding per unit increase of INR was not higher than the corresponding figure calculated for death from all causes. The risk of death was significantly higher for patients who reached INR levels of $\geqslant 3.0$ after an increase in anticoagulant dose compared with those who reached the same level spontaneously $(\mathrm{P}=0.047)$.

The target INR depended largely on the type of indication for anticoagulation rather than the individual patient's state of health. In some clinics the target value was the same for all patients. The target value made no significant difference to the risk of death associated with the actual INR.

There were 1069 deaths observed within seven weeks after an INR measurement of $\geqslant 3$.0. Using the Poisson model, we calculated the expected number of deaths to be 569 provided that the risk was put equal to that of an INR of 2.9. Thus, at least 500 deaths in our study were associated with, but not necessarily caused by, a high INR value. Rough estimates of the number of patients treated with oral anticoagulation in Sweden suggest that there were 65 deaths associated with high INR values per million inhabitants annually.

\section{Discussion}

Our results show that mortality among patients treated with oral anticoagulants was lowest at an INR value of 2.2-2.3 and that it increased substantially at higher INR levels. Thus, we confirm the beneficial effects of anticoagulants on thromboembolism when the INR is increased from 1.0 to 2.3 and confirm reports of therapeutic effects with INR values lower than 2.0.

The association between risk of bleeding and intensity of treatment is well known, as is the need for vigilance to avoid occurrence of high INR values. ${ }^{9-13}$ Our findings indicate that preventive measures must also be taken when a high INR has occurred. Hylek et al found that patients without symptoms at time of measurement of an INR above 6.0 faced a substantial risk of major haemorrhage in the next 14 days. ${ }^{14}$ Studies on the use of vitamin $\mathrm{K}$ after high INR values will be of interest.

As the frequency of autopsies was low in our study, we could not accurately determine the number of deaths due to bleeding even though we could accurately measure the increase in mortality with increasing INR. We expected that bleeding should be the only cause of death that increased with increasing INR, but our analysis of deaths from cerebral bleeding suggests that other causes dominated. As well as an indicator of high risk of bleeding, a high INR has been reported to be an indicator of a final stage of disease, and

Mortality from all causes and from bleeding as a main or contributing cause among outpatients of Swedish anticoagulation clinics by level of anticoagulation (measured as international normalised ratio (INR))

\begin{tabular}{|c|c|c|c|c|c|}
\hline \multirow[b]{2}{*}{ INR } & \multirow[b]{2}{*}{ Patient years } & \multicolumn{2}{|c|}{ No of deaths } & \multicolumn{2}{|c|}{ Mortality/1000 patient years } \\
\hline & & All causes & Bleedings & All causes & Bleedings \\
\hline-0.9 & 36.7 & 4 & 0 & 109.0 & 0 \\
\hline $1.0-1.4$ & 1020.3 & 125 & 6 & 122.5 & 5.9 \\
\hline 1.5-1.9 & 7476.3 & 450 & 22 & 60.2 & 2.9 \\
\hline $2.0-2.4$ & 24474.5 & 1036 & 66 & 42.3 & 2.7 \\
\hline $2.5-2.9$ & 17927.6 & 849 & 67 & 47.4 & 3.7 \\
\hline $3.0-3.4$ & 7052.7 & 479 & 42 & 67.9 & 6.0 \\
\hline $3.5-3.9$ & 1672.9 & 240 & 22 & 143.5 & 13.2 \\
\hline $4.0-4.4$ & 380.4 & 86 & 5 & 226.1 & 13.1 \\
\hline $4.5-4.9$ & 205.6 & 73 & 5 & 355.1 & 24.3 \\
\hline $5.0-5.9$ & 161.4 & 129 & 3 & 799.3 & 18.6 \\
\hline $6.0-6.9$ & 30.4 & 36 & 2 & 1184.2 & 65.8 \\
\hline $7.0-7.9$ & 25.8 & 26 & 3 & 1007.8 & 116.3 \\
\hline
\end{tabular}


several factors are known to influence INR, including malnutrition,${ }^{15}$ diarrhoea, ${ }^{16}$ malignancy,${ }^{17}$ serious comorbid diseases, ${ }^{17} 18$ and interaction of warfarin with other drugs. Another possible explanation is some synergistic disturbance in regulation of coagulation before a high INR. Jansson et al have found an increased mortality ${ }^{19}$ and increased frequency of bleeding ${ }^{20}$ associated with high levels of thrombomodulin during long term anticoagulation treatment. It is also possible that warfarin may have toxic effects: death from the rare side effect of skin necrosis has been reported. ${ }^{2}$

As a spontaneous increase of INR is often seen in moribund patients, it might be expected that patients with this phenomenon had the highest risk of death for a certain level of INR compared with others who reached the same level after an increased dose of anticoagulant. However, our study indicates that the risk of death was significantly higher among patients whose high INR followed an increased drug dose. This finding indicates that preventive actions can be taken. In order to avoid high INR values and risky elevations of doses there is a need for more careful treatment with oral anticoagulants. We propose the use of lower target values of INR and a smaller therapeutic window compared with what is used today. An INR of 2.2-2.3 seems to be associated with the lowest risk of death for all indications.

We thank Jan Ramebäck and Knut Fahlén for programming and organisation necessary for the data collection.

Contributors: MF performed the literature research and $\mathrm{AO}$ planned and made the statistical analyses. Both authors initiated the project and contributed to the writing, and both are guarantors for the study.

Competing interests: None declared.

1 Turpie AG. Safer anticoagulant therapy after heart valve replacement. Recommendations for less intense regimens. Postgrad Med 1997;101:85-6, 89-90, 93-4.

2 Hirsh J. Optimal intensity and monitoring warfarin. Am J Cardiol $1995 ; 75: 39-42 B$.

3 Stein PD, Alpert JS, Bussey HI, Dalen JE, Turpie AG. Antithrombotic therapy in patients with mechanical and biological prosthetic heart valves. Chest 2001;119:220-7S

4 Katircioglu SF, Yamak B, Ulus AT, Iscan HZ, Mavitas B, Tasdemir O. Aortic valve replacement with the St. Jude Medical prosthesis and fixed dose anticoagulation. J Card Surg 1997;12:363-70.

5 Katircioglu SF, Yamak B, Ulus AT, Tasdemir O, Bayazit K. Mitral valve replacement with St. Jude Medical prosthesis and low-dose anticoagulation in patients aged over 50 years [see comments]. J Heart Valve Dis 1998:7:455-9.

6 Moinuddeen K, Quin J, Shaw R, Dewar M, Tellides G, Kopf G, et al. Anticoagulation is unnecessary after biological aortic valve replacement. $\mathrm{Cir}$ culation 1998;98:II95-8.

\section{What is already known on this topic}

The optimal degree of anticoagulation (expressed as the international normalised ratio (INR)) for different indications is still unclear, but the increased risk of death due to bleeding at high INR values is well known

\section{What this study adds}

This large study of medical records from anticoagulation clinics in Sweden confirmed the substantial excess mortality at high INR values and indicated optimal treatment to be in a small therapeutic window with INR close to 2.2-2.3, irrespective of the indication for anticoagulant treatment

7 Breslow NE, Day NE. Statistical methods in cancer research Vol 2. Lyon: IARC, 1987.

8 Levine M, Hirsh J, Gent M, Arnold A, Warr D, Falanga A, et al Double-blind randomised trial of a very-low-dose warfarin for prevention of thromboembolism in stage IV breast cancer. Lancet 1994;343:886-9.

9 Acar J, Iung B, Boissel JP, Samama MM, Michel PL, Teppe JP, et al. AREVA: multicenter randomized comparison of low-dose versus standard-dose anticoagulation in patients with mechanical prosthetic heart valves [see comments]. Circulation 1996;94:2107-12

10 Altman R, Rouvier J, Gurfinkel E, D’Ortencio O, Manzanel R, de La FL, et al. Comparison of two levels of anticoagulant therapy in patients with substitute heart valves. J Thorac Cardiovasc Surg 1991;101:427-31.

11 Saour JN, Sieck JO, Mamo LA, Gallus AS. Trial of different intensities of anticoagulation in patients with prosthetic heart valves. $N$ Engl J Med 1990;322:428-32.

12 Tientadakul P, Opartkiattikul N, Sangtawesin W, Sakiyalak P. Effect of different oral anticoagulant intensities on prothrombin fragment $1+2$ in Thai patients with mechanical heart valve prostheses. J Med Assoc Tha 1997;80:81-6.

13 Turpie AG, Gunstensen J, Hirsh J, Nelson H, Gent M. Randomised comparison of two intensities of oral anticoagulant therapy after tissue heart valve replacement. Lancet 1988;i:1242-5.

14 Hylek EM, Chang YC, Skates SJ, Hughes RA, Singer DE. Prospective study of the outcomes of ambulatory patients with excessive warfarin anticoagulation. Arch Intern Med 2000;160:1612-7.

15 Lehmann AB. Vulnerability to warfarin: could undernutrition be a predictor? Arch Intern Med 1997;157:1385.

16 Smith JK, Aljazairi A, Fuller SH. INR elevation associated with diarrhea in a patient receiving warfarin. Ann Pharmacother 1999;33:301-4.

17 Chan A, Woodruff RK. Complications and failure of anticoagulation therapy in the treatment of venous thromboembolism in patients with disseminated malignancy. Aust N Z J Med 1992;22:119-22.

18 Landefeld CS, Beyth RJ. Anticoagulant-related bleeding: clinical epidemiology, prediction, and prevention. Am J Med 1993;95:315-28.

19 Jansson JH, Boman K, Brannstrom M, Nilsson TK. Increased levels of plasma thrombomodulin are associated with vascular and all-cause mortality in patients on long-term anticoagulant treatment. Eur Heart $J$ 1996;17:1503-5.

20 Jansson JH, Boman K, Brannstrom M, Nilsson TK. High concentration of thrombomodulin in plasma is associated with hemorrhage: a prospective study in patients receiving long-term anticoagulant treatment. Circulation 1997;96:2938-43.

21 DeFranzo AJ, Marasco P, Argenta LC. Warfarin-induced necrosis of the skin. Ann Plast Surg 1995;34:203-8.

(Accepted 1 August 2002)

\section{A memorable patient}

\section{A crucial examination}

The clinical examination of the MRCP was fast approaching. We had scoured the hospital for clinical signs, and almost every patient had kindly allowed us to listen on and prod at them in our well rehearsed, near choreographed examination routine. Would I still be able to execute the routine in a semi-autonomic fashion after the weekend? Panic struck.

I found the easiest "patient" available on a weekend at home was my dear wife. I reminded myself that, even though she was not a patient with clinical signs, I should perform the thorough routine as though it was the exam itself.

"Hmm. There seems to be a little tremor in both your hands," I said to my rather astonished wife, "and your pulse is rather fast for someone at rest." At this point she suggested that I must have been so inundated with the abnormal that I was unable to differentiate it from normality. But then came the cardiac flow murmur, weight loss, and the persistent heat intolerance that we had both previously thought insignificant.

Needless to say, blood tests confirmed an overactive thyroid gland, and I have learnt that the value of a thorough physical examination goes far beyond passing examinations.

Daren C Y Teoh senior house officer, Birmingham

We welcome articles up to 600 words on topics such as A memorable patient, A paper that changed my practice, My most unfortunate mistake, or any other piece conveying instruction, pathos, or humour. If possible the article should be supplied on a disk. Permission is needed from the patient or a relative if an identifiable patient is referred to. 